\title{
MÉTODOS ECOCARDIOGRÁFICOS PARA EVALUAR LA AURÍCULA DERECHA UTILIZANDO LOS VALORES DE CORTE PUBLICADOS POR SOCIEDADES INTERNACIONALES
}

\author{
ECHOCARDIOGRAPHIC METHODS TO EVALUATE THE RIGHT ATRIUM \\ USING THE VALUES PUBLISHED BY INTERNATIONAL SOCIETIES
}

\author{
MARÍA DEL PILAR ACOSTA ${ }^{1}$, MARCELO STORINO ${ }^{2}$, GUILLERMO GODOY ${ }^{2}$, VANESA MARASA ${ }^{2}$, VÍCTOR ARREGUI ${ }^{3}$, JUAN ERRIEST ${ }^{4}$, JORGE \\ CAMILLETTII ${ }^{5}$, ALEJANDRO VILCHEZ ${ }^{3}$, DIEGO MARTÍNEZ DEMARÍA ${ }^{3}$, MARÍA LAURA PLASTINO ${ }^{6}$
}

\section{RESUMEN}

Introducción. El tamaño de la aurícula derecha (AD) medido por ecocardiografía ha mostrado ser un marcador de riesgo cardiovascular en diferentes condiciones cardíacas. La cuantificación del tamaño de la AD se realiza más comúnmente en la vista apical de 4 cámaras, donde se realiza planimetría para estima el área. Hasta el momento, no se conocen estudios que correlacionen el área de la AD (Área AD) con los nuevos valores de corte propuestos para el volumen de la AD. Las guías de la Sociedad Americana de Ecocardiografía (ASE)/Sociedad Europea de Cardiología (ESC) establecen que el parámetro recomendado para evaluar el tamaño de la $\mathrm{AD}$ es el volumen $\mathrm{AD}(\mathrm{Vol} \mathrm{AD})$ calculado a través del método área-longitud o de sumatoria de discos en un solo plano apical de 4 cámaras. Objetivos. Correlacionar el volumen de la $\mathrm{AD}(\mathrm{Vol} \mathrm{AD})$ con el área de la $\mathrm{AD}$ según los parámetros de las recomendaciones ASE/ESC.

Materiales y métodos. Estudio observacional y descriptivo, retrospectivo, donde se analizaron 109 pacientes que concurrieron al laboratorio de Ecocardiografía del Hospital Italiano de La Plata para control cardiovascular entre los meses de septiembre y octubre del año 2015; la edad media fue de 56,41 \pm 17 años, 53,21\% de sexo masculino.

Resultados. Del total de participantes, 24 (22,01\%) fueron clasificados con AD anormal por Área AD y $15(13,76 \%)$ con $A D$ anormal por Vol $A D(p<0,02)$. De los 85 pacientes que tuvieron AD normal según Área AD, el 2,75\% (3 pacientes) tu vieron $\mathrm{V} v \mathrm{O}$ AD anormal; contrariamente, de los 24 que tuvieron $A D$ anormal por área, 12 tuvieron también AD anormal por Vvol AD (concordancia moderada, kappa 0,53). El análisis estadístico según género evidenció una concordancia mejor para el sexo femenino (kappa 0,73 vs. 0,43). La variabilidad interobservado para evaluar las dimensiones de la AD según Área AD fue de $2,4 \pm 0,2 \mathrm{~mm}$ y según Volvolumen AD de $4 \pm 0,36 \mathrm{ml} / \mathrm{m} 2$; valores que concuerdan con reportes previos. Se observó una correlación positiva lineal moderada entre el Área AD y el Vol AD $(r=0,799)$

Conclusión. Los valores de corte para tamaño AD según el área y el volumen han demostrado una buena concordancia, mejor aún para el sexo femenino. Es importante seguir las recomendaciones de las guías de la ASE/ESC, evaluar a los pacientes según los valores de corte recomendados y definir dilatación AD por Vol AD y Área AD.

Palabras clave: aurícula derecha, ecocardiografía.

\section{ABSTRACT}

Introduction. The size of the right atrium (RA) measured by echocardiography has been shown to be a marker of cardiovascular risk in different heart diseases. The quantification of the size of the RA is most commonly performed in the apical view of 4 chambers, where planimetry is performed to estimate the area. So far, there are no known studies that correlate the area of RA with the new proposed cut-off values for RA volume. The guidelines of the American Society of Echocardiography (ASE) / European Society of Cardiology (ESC) establish that the recommended parameter to evaluate the size of the RA is the RA volume calculated through the area-length method or the sum of discs in a single apical plane of 4 chambers.

Objective. To correlate the volume of the RA (RA vol) with the area of the RA according to the parameters of the ASE / ESC recommendations.

Materials and Methods. Observational and descriptive, retrospective study; 109 patients were analyzed who attended the Echocardiography laboratory for cardiovascular monitoring between the months of September and October of 2015; the average age was $56.41 \pm 17$ years, $53.21 \%$ were males.

Results. Of the total of participants, 24 (22.01\%) were classified with abnormal RA by RA area and $15(13.76 \%)$ with abnormal RA by RA Vol $(p<0.02)$. Of the 85 patients who had normal RA according to RA Area, 2.75\% (3 patients) had abnormal RA Vol. Conversely, of the 24 who had abnormal RA by area, 12 also had abnormal RA by RA Vol (moderate agreement, kappa 0.53). The statistical analysis ac cording to gender showed a better agreement for the female sex (kappa 0.73 vs 0.43). Interobserver variability to evaluate the dimensions of the RA according to RA Area was $2.4 \pm 0.2 \mathrm{~mm}$ and according to RA volume of $4 \pm 0.36 \mathrm{ml} / \mathrm{m} 2$; values that agree with previous reports. A moderate linear positive correlation was observed between RA Area and RA Vol (r 0.799).

Conclusion. The cut-off values for RA size according to area and volume have shown a good agreement, even better for the female sex. It is important to foIlow the recommendations of the ASE / ESC guidelines, evaluate patients according to the recommended cut-off values and define RA dilatation by RA Vol and RA area.

Keywords: right atrium, echocardiography

REVISTA CONAREC 2018;33(145):180-182 | DOI:10.32407/RCON/2018145/0180-0182

\footnotetext{
1. Jefa de Residentes de Cardiología.

2. Médico staff de la Unidad de Eco Doppler Cardiovascular.

3. Médico staff del Área de Cardiología.

4. Jefe de Medicina Nuclear.

5. Jefe del Área de Cardiología.

6. Jefa de la Unidad de Eco Doppler Cardiovascular.
}

Cátedra de Posgrado en Cardiología, Facultad de Ciencias Médicas, Universidad Nacional de La Plata. Hospital Italiano de La Plata. La Plata, provincia de Buenos Aires, Rep. Argentina.

$\triangle$ Correspondencia: Dra. María del Pilar Acosta. Calle $9 n^{\circ} 1124$ 2B, CP 1900, La Plata. Buenos Aires, Rep. Argentina. madelpilar_acosta@hotmail.com

Los autores declaran no poseer conflictos de intereses.

Recibido: 10/10/2017 | Aceptado: 01/06/2018

\section{INTRODUCCIÓN}

La aurícula derecha (AD) contribuye con el llenado ventricular derecho, inicialmente como reservorio del retorno venoso sistémico, luego como conducto pasivo en la diástole temprana y finalmente como conducto activo en la diástole tardía durante la contracción auricular'. El tamaño de la AD medido por ecocardiografía ha demostrado ser un marcador de riesgo cardiovascular en diferentes condiciones cardíacas, presenta remodelado reverso luego de la ablación por fibrilación auricular y pareciera ser dependiente del genero². El Área AD es predictor de mortalidad o trasplante en pacientes con hipertensión pulmonar primaria ${ }^{3,4}$. Utilizando el ecocardiograma, la AD se puede 


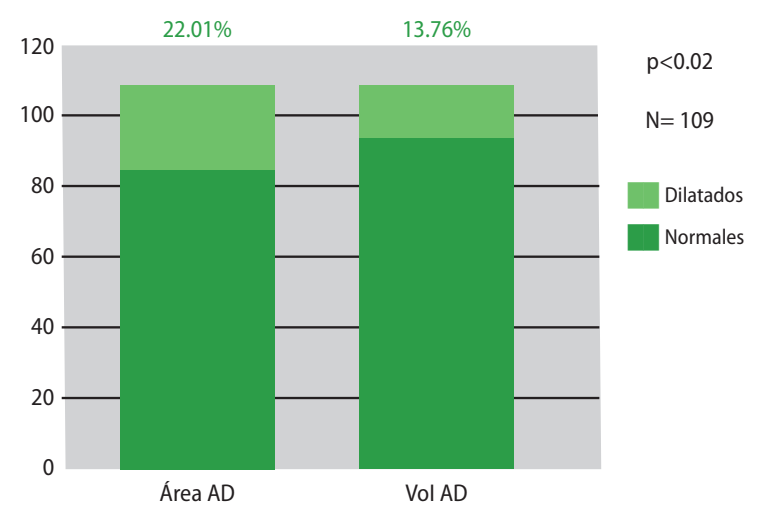

Figura 1. Distribución de pacientes con aurícula derecha dilatada según el método de evaluación ecocardiográfico utilizado. AD: aurícula derecha. Vol: volumen

apreciar desde diferentes vistas; la cuantificación del tamaño de la AD se realiza más comúnmente en la vista apical de 4 cámaras. Desde esta ventana se realiza planimetría para estimar el área (límite superior normal $\left.18 \mathrm{~cm}^{2}\right)^{5-7}$.

Los volúmenes de la AD son más sólidos y precisos para la determinación del tamaño de la AD en comparación con las dimensiones lineales. Se ha propuesto para la determinación del Vol AD una vista única de área longitud y/o técnica de sumatoria de discos ${ }^{6,8}$.

Hasta el momento no se conocen estudios que correlacionen el Área $\mathrm{AD}$ con los nuevos valores de corte propuestos para el Vol AD.

\section{OBJETIVO}

El propósito de este estudio es correlacionar el volumen de la AD con el área de la $A D$, según los parámetros de las recomendaciones de la Sociedad Americana de Ecocardiografía (ASE) y la Sociedad Europea de Cardiología (ESC).

\section{MATERIALES Y MÉTODOS}

En el presente estudio observacional y descriptivo, retrospectivo, se analizaron 109 pacientes que concurrieron al laboratorio de Ecocardiografía del Hospital Italiano de La Plata para control cardiovascular entre los meses de Septiembre y Octubre del año 2015. El trabajo fue previamente evaluado y aceptado por el comité de bioética del Hospital Italiano de La Plata.

\section{POBLACIÓN Y DATOS ECOCARDIOGRÁFICOS}

A los participantes se les realizó eco-Doppler cardíaco convencional transtorácico utilizando un ecocardiógrafo General Electric Vivid S5.

Todas las mediciones se realizaron de acuerdo con las recomendaciones actuales de la ASE y ESC.

Se incluyeron mediciones de diámetro ántero-posterior de aurícula izquierda (diám APAl), área de la aurícula izquierda (Área Al) y volumen auricular izquierdo indexado a la superficie corporal (VolAli). El Vol AD fue evaluado a través del método de área longitud y/o técnica de sumatoria de discos desde una vista única en 4 cámaras. La medida se obtuvo al final de la sístole, en el frame que precede la apertura de la válvula tricúspide. Se trazó la planimetría de la AD y



Figura 2. Correlación entre el área y el volumen de la aurícula derecha. AD: aurícula derecha. Vol: volumen.

el volumen fue indexado a la superficie corporal. El Área AD fue medida en vista apical de 4 cámaras y en fin de sístole, en el cuadro justo antes de la apertura de la válvula tricúspide, mediante el trazado de la interfaz sangre tejido de la AD, excluyendo el área debajo del anillo de la válvula tricúspide.

Los diámetros del ventrículo izquierdo (VI) fueron medidos con modo $\mathrm{M}$ en eje corto paraesternal izquierdo, guiado por 2D. La función sistólica del VI fue evaluada con la fracción de eyección (FEy) a través del método de Simpson. Se obtuvo el índice de masa VI (IMVI) según las recomendaciones de la ASE.

Cada valor registrado representa el promedio de tres latidos consecutivos. Todas las medidas fueron realizadas online y grabadas en una base de datos.

Para evaluar la variabilidad interobservador, dos operadores independientes, midieron el área y el volumen de la AD en 10 pacientes seleccionados, sin conocer los resultados obtenidos por el otro observador.

\section{TAMAÑO AD}

Las guías ASE/ESC establecen que el parámetro recomendado para evaluar el tamaño de la AD es el Vol AD calculado a través del método área-longitud o de sumatoria de discos en un solo plano apical de 4 cámaras. El rango normal del volumen de la AD por Eco $2 \mathrm{D}$ es de $25 \pm 7 \mathrm{ml} / \mathrm{m}^{2}$ en hombres y $21 \pm 6 \mathrm{ml} / \mathrm{m}^{2}$ en mujeres ${ }^{9}$.

\section{DATOS CLÍNICOS}

Al momento del examen se registraron edad, altura, peso, ritmo cardíaco y comorbilidades.

\section{ANÁLISIS ESTADÍSTICO}

Los resultados fueron expresados como porcentajes o medias con desvío estándar (DE). Para evaluar la fuerza de relación entre el área y el volumen de la AD, y calcular la asociación entre estas variables, se utilizó el coeficiente de correlación de Pearson (r). El test de concordancia de kappa se utilizó para calcular la fuerza de acuerdo entre ambos métodos.

La variabilidad interobservador se expresó como la media y DE de la diferencia absoluta entre las medidas realizadas por dos investigadores. La significación estadística de referencia fue $p<0,05$. El análisis fue realizado utilizando Microsoft Excel. 


\section{RESULTADOS}

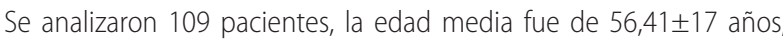
$53,21 \%$ de sexo masculino.

\section{ECO DOPPLER CARDÍACO Y CORRELACIÓN ENTRE ÁREA AD Y VOL AD}

Al utilizar los valores de corte publicados para agrandamiento de la AD, 24 pacientes (22,01\%) fueron clasificados con AD anormal por Área AD y $15(13,76 \%)$ con $A D$ anormal por Vol $A D(p<0,02)$ (Figura 1).

De los 109 pacientes analizados, se observó una correlación positiva lineal moderada entre el Área AD y el Vol AD $(r=0,799)$ (Figura 2). De los 85 pacientes que tuvieron AD normal según Área AD, el 2,75\% (3 pacientes) tuvo Vol AD anormal, contrariamente, de los 24 que tuvieron $A D$ anormal por área, 12 tuvieron también $A D$ anormal por Vo AD (concordancia moderada, kappa 0,53).

El análisis estadístico según género evidenció que la concordancia fue mejor para el sexo femenino en relación con el masculino (kappa 0,73 vs. 0,43)

\section{VARIABILIDAD INTEROBSERVADOR}

La variabilidad interobservador para evaluar las dimensiones de la $A D$

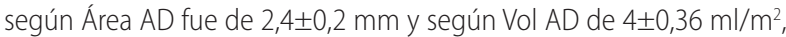
valores que concuerdan con reportes previos.

\section{DISCUSIÓN}

La evaluación del tamaño auricular derecho medida a través del Vol AD y Área AD, aplicando los valores de corte publicados, permiten estratificar el riesgo en un significativo número de pacientes.

Como la AD no se agranda en forma simétrica, la evaluación unidimensional, sería poco sensible para detectar los cambios en el tamaño de la AD. Nuestros resultados, confirman hallazgos previos y demuestran que en esta población se pone de manifiesto que al utilizar los valores de corte para identificar pacientes con dilatación auricular derecha deberían considerarse las dimensiones obtenidas a través del Vol $A D$ y Área $A D^{8}$.

En relación con otros estudios, el presente trabajo demostró también que el tamaño AD depende del genero ${ }^{10,11}$.

\section{CONCLUSIÓN}

Los valores de corte para el tamaño AD según el área y el volumen han demostrado una buena concordancia, mejor aún para el sexo femenino. Es importante seguir las recomendaciones de las guías de la ASE/ESC, evaluar a los pacientes según los valores de corte recomendados y definir dilatación $A D$ por Vol $A D$ y Área AD.

\section{BIBLIOGRAFÍA}

1. Gaynor SL, Maniar HS, Prasad SM, Steendijk P, Moon MR. Reservoir and conduit function of right atrium: impact on right ventricular filling and cardiac output. Am J Physiol Heart Circ Physiol 2005;288(5):H2140-5.

2. Pathak RK, Middeldorp ME, Lau DH, Mehta AB, Mahajan R, et al. Aggressive risk factor reduction study for atrial fibrillation and implications for the outcome of ablation: the ARREST-AF cohort study. I Am Coll Cardiol 2014;64(21):2222-31.

3. Galiè N, Humbert M, Vachiery JL, Gibbs S, Lang I, Torbicki A, et al. 2015 ESC/ERS Guidelines for the diagnosis and treatment of pulmonary hypertension: The Joint Task Force for the Diagnosis and Treatment of Pulmonary Hypertension of the European Society of Cardiology (ESC) and the European Respiratory Society (ERS). Endorsed by: Association for European Paediatric and Congenital Cardiology (AEPC), International Society for Heart and Lung Transplantation (ISHLT). Eur Heart J 2016:37(1):67-119.

4. Lescano AJ, Gomes Sánchez MA. Hipertensión pulmonar. lera Ed. Buenos Aires: Librería AKADIA Editorial, 2017.

5. Rudski LG, Lai WW, Afilalo J, Hua L, Handschumacher MD, Chandrasekaran K, et al. Guidelines for the echocardiographic assessment of the right heart in adults: a report from the American Society of Echocardiography endorsed by the European Association of Echocardiography, a registered branch of the European Society of Cardiology, and the Canadian Society of Echocardiography. J Am Soc Echocardiogr 2010;23(7):685-713.
6. Kou S, Caballero L, Dulgheru R, Voilliot D, De Sousa C, Kacharava G, et al. Echocardiographic reference ranges for normal cardiac chamber size: results from the NORRE study. Eur Heart J Cardiovasc Imaging 2014;15(6):680-90

7. Quraini D, Pandian NG, Patel AR. Three-dimensional echocardiographic analysis of right atrial volume in normal and abnormal hearts: comparison of biplane and multiplane methods. Echocardiography 2012;29(5):608-13.

8. D'Oronzio U, Senn O, Biaggi P, Gruner C, Jenni R, Tanner FC, et al. Right heart assessment by echocardiography: gender and body size matters. J Am Soc Echocardiogr 2012;25(12):1251-8

9. Lang RM, Badano LP, Mor-Avi V, Afilalo J, Armstrong A, Ernande L, et al. Recomendaciones para la Cuantificación de las Cavidades Cardíacas por Ecocardiografía en Adultos: Actualización de la Sociedad Americana de Ecocardiografía y de la Asoci ación Europea de Imagen Cardiovascular. J Am Soc Echocardiogr 2015:28(1):1-39.

10. Lang RM, Bierig M, Devereux RB, FlachskampfFA, Foster E, Pellikka PA, et al. Recommendations for chamber quantification: a report from the American Society of Echocardiography's Guidelines and Standards Committee and the Chamber Quantification Writing Group, developed in conjunction with the European Association of Echocardiography, a branch of the European Society of Cardiology. J Am Soc Echocardiogr 2005; 18(12): 1440-63.

11. Peluso D, Badano LP, Muraru D, Dal Bianco L, Cucchini U, Kocabay G, et al. Righ atrial size and function assessed with three-dimensional and speckle-tracking echocardiography in 200 healthy volunteers. Eur Heart J Cardiovasc Imaging 2013:14(11):1106-14 